\title{
EL MANEJO DE LOS TURNOS DE HABLA: APLICACIONES DEL ANÁLISIS DE LA CONVERSACIÓN EN LA ENSEÑANZA DEL ESPAÑOL COMO SEGUNDA LENGUA
}

\author{
Patricia Guillén Solano
}

\begin{abstract}
RESUMEN
Este artículo analiza el manejo de los turnos de habla por parte de una hablante no nativa del español durante una conversación con hablantes nativos, partiendo de la premisa de que los diferentes mecanismos de tomar o ceder la palabra, así como los diferentes papeles comunicativos que asume el hablante deben ser estudiados en el aula de español como segunda lengua, pues constituyen parte fundamental del desarrollo de la competencia comunicativa.

Palabras clave: turno de habla, competencia comunicativa, conversación, papel comunicativo, español como segunda lengua.
\end{abstract}

\begin{abstract}
This article analyses turn taking strategies used by a non native Spanish speaker during a conversation with native speakers. This study is based on the premise that the different turn taking mechanisms as well as the different communicative roles assumed by the speaker must be studied in the Spanish as a second language class, since they play a fundamental role in communicative competence development.

Key words: turn taking, communicative competence, conversation, communicative role, Spanish as a second language.
\end{abstract}

\section{Introducción}

Para Calsamiglia y Tusón, la conversación espontánea es "la forma primera, primaria y universal de realización de la oralidad; la forma más característica en que las personas se relacionan y llevan a cabo sus actividades cotidianas como seres sociales" (1999: 32). Partiendo de esta premisa, queda claro que los enfoques de enseñanza de segundas lenguas basados en

ML. Patricia Guillén Solano. Profesora. Escuela de Filología, Lingüística y Literatura. Universidad de Costa Rica.

Correo electrónico: patrihauster@gmail.com

Recepción: 15- 02- 2011

Aceptación: 22- 03- 2011 
nociones comunicativas y funcionales deben rescatar la importancia de incorporar en las aulas el análisis de la conversación. De hecho, el término competencia comunicativa, acuñado por Gumperz y Hymes a partir de sus estudios sobre etnografía de la comunicación ${ }^{1}$, se define como "aquello que un hablante necesita saber para comunicarse de manera eficaz en contextos socialmente significantes" (1972: 7). El concepto se profundiza al relacionarse con la habilidad que cada hablante posee para actuar, pues no es suficiente que sepa sobre la lengua, sino que, mediante ella, logre realizar actos para conseguir sus objetivos en situaciones particulares.

También, los planteamientos de la pragmática enfatizan la importancia de partir del uso real del lenguaje, "de modo que el sistema formal no puede desligarse de los contextos de uso, de la identidad individual y social de los interlocutores, de sus intenciones y de su conocimiento y manera de relacionarse con el mundo" (Grande Alija 2005: 332).

En este uso real que se manifiesta, precisamente, en la conversación, ubicamos el tema de nuestro estudio, los cambios de turno. A pesar de su importancia, es común que no suelan tratarse de forma explícita en clase. Sobre este particular, Brenes Peña (2010: 734) señala que "el desconocimiento de las reglas que rigen la alternancia de turnos en cada género y situación comunicativa puede originar errores pragmáticos que afectan negativamente el desarrollo del proceso comunicativo".

Ambjoern (2008), por su parte, hace hincapié en la presentación desproporcionada que se hace del discurso oral monológico en el aula de lenguas extranjeras: exposiciones, narraciones guiadas y preguntas dirigidas por el profesor, actividades que, comúnmente, están vinculadas a un texto en particular. Debido a ello, plantea la necesidad de que la competencia comunicativa conduzca a la competencia conversacional, entendida como "la capacidad de participar en una comunicación bilateral o multilateral donde la distribución de turnos es administrada libremente por las partes, que se alternan en la posesión de la palabra” (2008: 3).

El propósito de este artículo consiste en analizar, a la luz del desarrollo de la competencia comunicativa, el manejo de los turnos de habla por parte de una hablante no nativa del español, partiendo de la premisa de que los diferentes mecanismos para tomar o ceder la palabra deben ser estudiados en el aula de español como segunda lengua.

\section{La conversación espontánea y el cambio de turnos}

La conversación implica un número relativamente restringido de participantes que se caracterizan por no tener papeles predeterminados. Presenta además un carácter familiar e improvisado, en tanto "los temas que se abordan, la duración del intercambio o el orden de los turnos de palabra se determina paso a paso, de forma relativamente libre" (Kerbrat-Orecchioni 1996: 8).

Briz (1998: 42) caracteriza la conversación, frente a otro tipo de discurso hablado, con los siguientes rasgos:

a) Una interlocución en presencia, conversación cara a cara.

b) Inmediata, actual (aquí y ahora).

c) Con toma de turno no predeterminada.

d) Dinámica, con alternancia de turnos inmediata.

e) Cooperativa en relación con el tema de conversación y la intervención del otro.

La conversación espontánea presenta, en general, una planificación sobre la marcha, tiene una finalidad interpersonal (un fin comunicativo socializador) y se manifiesta en un 
tono informal, no especializado. La alternancia de turnos no predeterminada es uno de los rasgos que, precisamente, la definen. El turno, entendido como "un hueco estructural rellenado con emisiones informativas que son reconocidas por los interlocutores mediante su atención manifiesta y simultánea" (Briz 2000: 14) permite que la conversación progrese dentro de un orden. Siguiendo a este autor, "desde el punto de vista conceptual, el turno responde a una forma de sucesión establecida o prevista para hacer, decir o recibir algo. Es un mecanismo de regulación social en cualquier interacción" (10).

A diferencia de otros autores, para Briz un cambio de voz o intervención no implica necesariamente un cambio de turno, puesto que "habrá un nuevo turno y, por tanto, alternancia de turno cuando la intervención reactiva de otro hablante B sea solicitada o, simplemente, reconocida y aceptada como tal por A" (14). De esta forma, la intervención debe ser reconocida, aceptada y ratificada por al menos un interlocutor para considerarse como un verdadero cambio de turno, pues se parte de que la conversación se basa en estrategias de negociación e intercambio.

Cada una de las emisiones que rellenan los turnos se denominan intervenciones: un enunciado o conjunto de enunciados (actos de habla) que emite un interlocutor, que puede ser de inicio (intenta provocar habla posterior) o de reacción.

De acuerdo con Calsamiglia y Tusón, (1999: 33) los mecanismos por los cuales se rige el cambio de turno son, básicamente, dos:

a) La heteroselección, que consiste en que quien está usando la palabra selecciona al siguiente hablante.

b) La autoselección, que consiste en que una de las personas presentes empieza a habla sin que quien tiene la palabra la haya seleccionado.

Las autoras señalan que, normalmente, estos mecanismos funcionan relativamente bien porque los interlocutores reconocen lo que se denomina lugares apropiados para la transición (LAT). Un LAT puede estar señalado por una pregunta, por rasgos como la entonación y las pausas, e incluso, por un gesto.

Cestero (2000) clasifica las alternancias de turnos en dos tipos, propias e impropias. Las primeras se producen una vez finalizado el turno de habla y sin superposición. Las segundas no cumplen con ninguno de estos requisitos y amenazan, por lo tanto, los dos principios básicos sobre los que se sustenta una conversación: el de cooperación y el de secuenciación.

El principio de cooperación se basa en que los participantes deben marcar el carácter del turno que poseen y su final, así como respetar las señales que el interlocutor les ofrece. Por su parte, el principio de secuenciación consiste en que el interlocutor debe marcar la relación que el nuevo turno puede tener con el turno anterior.

Dentro de la dinámica de los cambios de turno, se reconoce un tipo de intercambio muy importante, el par adyacente. Pares adyacentes típicos de la conversación son, por ejemplo, pregunta/respuesta, invitación/ aceptación-rechazo.

Para analizar esta dinámica dentro del desarrollo de la competencia comunicativa en español como segunda lengua, utilizaremos el modelo propuesto por Calsamiglia y Tusón (1999: 64), basado en la dimensión interlocutiva ${ }^{2}$, que analiza cómo se organiza la interacción y tiene en cuenta el espacio interactivo ocupado (el capital verbal), la manera de tomar la palabra y de pasar de un turno al siguiente y la forma en que los diferentes participantes asumen papeles comunicativos particulares. 
Esta dimensión se encuentra conformada por los siguientes elementos:

a) Capital verbal: número de tomas de palabras, número de palabras y tiempo ocupado.

b) Origen de las tomas de palabra: autoselección, heteroselección.

c) Modos de transición: pausa, solapamiento e interrupción.

d) Papeles comunicativos: aserción, pregunta, validación, demanda de validación, respuesta y gestión de los turnos de palabra, así como de los tema.

\section{La conversación coloquial y el cambio de turnos en español como segunda lengua}

Albelda y Fernández (2006) defienden la necesidad de incorporar el registro coloquial en la enseñanza del español como segunda lengua y, en particular, subrayan la importancia de analizar la lengua a partir de transcripciones de conversaciones orales espontáneas, pues estas incluyen muchos aspectos de la lengua hablada que no suelen tomarse en cuenta en materiales creados ad hoc.

Las autoras sugieren familiarizar a los estudiantes con los códigos propios de la transcripción, detallar los rasgos situacionales en que se lleva a cabo la conversación y enumerar los temas que trata. También, proponen una serie de actividades para trabajar distintos aspectos de una conversación, como lo son el uso de marcadores discursivos e intensificadores, la identificación de los rasgos del discurso informal y el llamado robo de turnos. Sobre este aspecto en particular, la actividad propuesta se dirige a reconocer diferentes estrategias para robar el turno. Destacan que los estudiantes deben saber cómo en la cultura comunicativa española se tiende a interrumpir o hablar simultáneamente, por ejemplo, y para ello es útil que identifiquen los mecanismos para apoderarse del turno. Asimismo, proponen el estudio de los turnos colaborativos, es decir, de las estrategias para apoyar lo dicho por el interlocutor.

Brenes Peña (2010) realiza un estudio en el cual plantea la importancia de abordar de forma explícita en el aula de segundas lenguas conceptos como cambio de turno, solapamiento e interrupción, ya que la total ausencia de estos elementos le puede restar naturalidad a la interacción, al tiempo que su uso inadecuado puede provocar un ambiente comunicativo marcado por la descortesía y la lucha por el turno de habla, de manera que resulta indispensable que los aprendices identifiquen en qué circunstancias comunicativas se admiten estos mecanismos y las metas interlocutivas que persiguen.

Por su parte, Ambjoern (2008) también propone la enseñanza explícita y sistemática de las estrategias involucradas en la comunicación interactiva. Destaca, a su vez, que esta perspectiva requiere la elaboración de material y actividades dirigidas a este fin, así como "la introducción de formas de entrenamiento lingüístico menos tradicionales" (4).

García (2005) elabora un estudio de la toma de palabra, la conexión entre turnos y la caracterización del turno en marcha en conversaciones de hablantes no nativos. En él, concluye que los hablantes no nativos presentan problemas para tomar la iniciativa en el manejo de la conversación y, en general, prefieren tomar el turno en un lugar apropiado para la transición antes que interrumpir. Tampoco se atreven a completar turnos si previamente no se han producido señales de vacilación. En cuanto al tema(s) de conversación, siempre les resulta más fácil seguir con el discurso ya trazado que introducir un tema nuevo, cambiar o dar por concluido uno anterior. 


\section{Análisis de la conversación entre hablantes nativos y no nativos: los turnos de habla}

En la conversación que sometemos a análisis, participan tres hablantes nativos del español y una hablante no nativa, cuya lengua materna es el japonés y que ha vivido y estudiado español en Costa Rica por ocho meses.

La conversación se da en una casa de habitación y tiene como finalidad establecer lazos sociales más estrechos entre los participantes. Todos los participantes tienen edades similares, entre 25 y 35 años. Los tres hablantes nativos son costarricenses, dos hombres y una mujer. Cabe destacar que la grabación se realizó secretamente (con consentimiento informado a posteriori), con el fin de propiciar una conversación lo más espontánea posible.

Para la transcripción, se siguen los lineamientos de Calsamiglia (1999: 360) en cuanto al uso de ortografía estándar, no utilización de mayúsculas (salvo en nombres propios) ni signos de puntuación. En lo que respecta a los códigos utilizados, se aplican los siguientes parámetros:

a) Las cifras a la izquierda del texto indican el número de la toma de palabra.

b) Las iniciales en mayúscula identifican a cada participante: HNN: hablante no nativo, HN1: hablante nativo 1, HN2: hablante nativo 2 y HN3: hablante nativo 3.

c) / // // indican pausas de diferente duración.

d) [ ] alrededor de un fragmento indica que esa secuencia se solapa con las palabras de otro participante, que se reproducen también entre corchetes. También se utilizan para marcar fenómenos no léxicos, tanto vocales como no vocales, por ejemplo [risas], [mirando a B].

e) $==$ al comienzo de una toma de palabra indica que no existe pausa entre esa toma $\mathrm{y}$ la anterior.

\subsection{Transcripción}

1. HN2: ¿y tiene hermanos menores / Yayoi?

2. HNN: sí / pero es cierto que ella / mi hermana / es más guapa que yo

3. HN1: = = ¿más? ///

4. HNN: creo / porque I de una parte / creo que los hermanos o hermanas menores son más guapos y guapas que [el primero o primera]

5. HN2: [no Yayoi porque] yo soy la menor [y no]

HN132: [yo eso lo sabía]

HN2: [ah sí]

6. HN1: yo sí [soy lindo]

7. HN2: [no pero usted es mayor]

8. HN1: ¿ah? /

9. HN2: Yayoi está diciendo que los mayores son más feos que los menores /

10. HN1: ah / ahora me está diciendo feo [Risas] no no /

dejémoslo en que yo soy lindo / 
11. HN3: imagínese / flaco viejo y feo [Risas]

12. HN1: no se burle mae //

mae ¿usted tiene papel aluminio? / ¿me regala un pedacito? /

es que este pedacito / este poquito que dejó ella / va para el taxi [Risas] me lo voy a comer en el taxi [Risas] yo me lo como en el taxi ///

13. HN2: ah sí / pero yo soy la menor y mi hermana es mucho más bonita que yo ///

14. HNN: ¿sí? / en Japón generalmente los menores son más atractivos / bonitos más como // como / generosas

HN3: = = no / pero yo creo que

15. HN2: = = tal vez, perdón / tal vez gastan más /

¿no es eso? ///

16. HNN: gastan más

HN3: = = no pero yo pienso que

17. HN2: = = y como son los menores los papás les dan más cosas / entonces se pueden comprar tal vez más ropa

18. HN1: = = sí / eso sí es típico porque si usted es mayor y usted tiene un hermano menor usted lo chinea más porque es su hermano menor / el más chiquito de la casa / eso pasa mucho / mi hermano me dice a mí quiero un carro y al día siguiente le compro el carro / porque son cosas que no le dieron a uno /

19. HN3: sí/ pero yo pienso que en todas las culturas siempre las personas más jóvenes siempre son como // no más cariñosas / sino como que a la gente les atraen más ///

20. HNN: ah ///

21. HN3: en todas las culturas es así / es como digamos aquí en Latinoamérica también alguien que tenga veinticinco o treinta años les gustan las muchachas de dieciocho o diecisiete [entonces yo creo]

22. HN2: [bueno es porque la generación] también va cambiando /

23. HNN: sí sí ///

24. HN2: ¿y tu hermana es estudiante? //

25. HNN: no, no / ella está trabajando / trabaja en una compañía que se alquilan las máquinas como de copiado copia // o algo así / no sé / las máquinas de oficina // no sé // yo no conozco bien / pero algunas máquinas // porque allá en Japón la compañía se alquila muchas cosas / muchas cosas que necesitan en cada compañía // como copiadora, fax o equipos de oficina /

26. HN3: ah / entonces hay compañias que alquilan el equipo de oficina /

27. HNN: sí //

28. HN3: aquí la compañía compra el equipo de oficina /

29. HNN: pero allá se alquilan 
30. HN2: = = pero también seguro allá es como cambia tan rápido /

31. HNN: sí como cambia // cuando ya podemos inmediatamente cambiamos

32. HN1: = = es más práctico que comprar la máquina

33. HN3: = sobre todo si la tecnología cambia hay unas que son más rápidas otras//

34. HNN: no sé bien sobre tecnología de Japón //

35. HN3: yo quisiera ir a Japón un año / desde Hokaido hasta Okinawa /

36. HNN: ¿por qué Hokaido? Okinawa sí / pero Hokaido /

37. HN3: porque es lo que está más al norte

38. HN2: = =ipero no es lo que está más al norte o sí?/

39. HNN: síl

40. HN3: bueno / yo no conozco la nieve

41. HN2: = = pero perdón ¿verdad que en Japón no podés hacer el recorrido en bicicleta?/

42. HNN: ¿en bicicleta? / ¿cuál estación? ¿en bicicleta? / ¿en dónde?

43. HN2: = = como cruzar el país // en verano tal vez.

44. HNN: en verano sí puede ser // porque yo creo / porque cada camino es muy seguro comparado aquí I

45. HN1: ¿pero para qué en bicicleta? / nada más agarra el tren magnético

46. HN2: = = sí / pero el tren es muy caro / yo cuando fui de Tokio a Nara eran como cien dólares casi //

47. HNN: sí sí / es verdad / transportes son muy caros //

48. HN2: ir de Japón a Tailandia eran doscientos dólares / era la mitad de ir en tren /

49. HNN: sí sí /

50. HN2: el tren bala costaba la mitad del avión /

51. HNN: sí // hay mucha competencia de compañías de avión

52. HN2: = = entonces cuando yo regresé a Tokio / yo fui en bus /

53. HNN: ah / sí sí / es buena idea /

54. HN1: ¿esos buses son acolchonaditos y acomodados al cuerpo?/

55. HN2: no [igual que los de]

56. HN3: [¿como los de Nicaragua?]

57. HN2: no no /

58. HN3: ¿como los de Hatillo? /

59. HN2: como los del aeropuerto / bien pero tampoco muy cómodos /// 


\subsection{Resultados}

De acuerdo con Calsamiglia y Tusón, “quién toma la palabra, cuántas veces, de qué manera y cuánto tiempo ocupa a lo largo de la interacción, aporta información muy clara y valiosa sobre los papeles comunicativos que adopta cada participante" (1999: 35).

Basándonos en esta afirmación, procederemos a analizar los resultados y a plantear los papeles comunicativos que asume cada hablante.

\section{Cuadro 1. Resultados}

\begin{tabular}{|c|c|c|c|c|}
\hline Participantes & Cantidad de turnos & $\begin{array}{c}\text { Origen de la toma } \\
\text { de turnos }\end{array}$ & $\begin{array}{c}\text { Cantidad de palabras } \\
\text { por hablante }\end{array}$ & Tiempo en minutos \\
\hline HNN & 19 & $\begin{array}{c}5 \text { autoselección } \\
14 \text { heteroselección }\end{array}$ & 190 & 3 \\
\hline HN1 & 9 & $\begin{array}{c}7 \text { autoseleección } \\
\text { 2 heteroselección }\end{array}$ & 147 & 1 \\
\hline HN2 & 20 & $\begin{array}{c}14 \text { autoselección } \\
6 \text { hereroselección }\end{array}$ & 198 & 1.5 \\
\hline HN3 & 11 & $\begin{array}{c}9 \text { autoselección } \\
4 \text { heteroselección }\end{array}$ & 139 & 1 \\
\hline
\end{tabular}

La hablante no nativa, como podemos ver, casi iguala (19 y 20, respectivamente) la cantidad de turnos de uno de los hablantes nativos. Junto con la HN2, es quien toma la palabra la mayor cantidad de veces. El HN1 y el HN3 están muy parejos en cuanto a sus participaciones (9 y 11, respectivamente).

En cuanto al número de palabras, quienes más turnos toman en la conversación (HNN y HN2) tienen, a su vez, la mayor cantidad de palabras (190 y 198, respectivamente). El HN1 supera solamente en 8 palabras al HN3.

El tiempo de cada participante muestra que aún cuando la HN2 y la HNN comparten un número similar de turnos, y la cantidad de palabras de la HN2 supera solo por 8 palabras al de la HNN, esta última ocupa el doble del tiempo. Esto se debe a la cantidad de pausas en su discurso. El HN1 y HN3 están muy parejos en cuanto al tiempo utilizado (1 minuto cada uno, aproximadamente).

En cuanto al origen de las tomas de turno, es relevante destacar que de 19 turnos de la HNN, sólo 5 corresponden a la autoselección, pues en el resto de sus intervenciones es quien está hablando quien le cede el turno (heteroselección), ya sea dirigiéndole una pregunta (par adyacente), o bien, mediante una señal no verbal, dirigiéndole una mirada. La hablante utiliza en cuatro ocasiones la autoselección para validar las ideas expresadas por otro hablante y en una ocasión para hacer una pregunta relacionada con la intervención anterior.

Por su parte, la estrategia preferida por los hablantes nativos es la autoselección: el HN1 utiliza la autoselección en 7 de sus 9 turnos. La HN2 en 14 de sus 20 participaciones y el HN3 en 9 de sus 13 intervenciones.

En los modos de transición, la HNN se caracteriza por el uso de la pausa, mientras que encontramos, en los tres hablantes nativos, casos de solapamiento (de 1 a 3 en cada hablante) y 3 robos de turno por parte de la HN2, los cuales, en dos ocasiones, son introducidos por la palabra perdón. 
Los papeles comunicativos, a su vez, dan cuenta de que los tres hablantes nativos, partiendo de la autoselección, son quienes gestionan los turnos y los temas, ya sea por medio de preguntas o demandas de validación. Por su parte, la HNN se limita a responder y a validar las afirmaciones e intervenciones de los HN y sólo gestiona 5 de sus 19 turnos.

\section{Conclusiones}

Partiendo de que el análisis del discurso es el análisis de la lengua en su uso (Brown y Yule 1993: 19), es importante, en el caso del español como segunda lengua y su enseñanza, incorporar este tipo de análisis si hemos de ser consecuentes con la propuesta de enfatizar el desarrollo de la competencia comunicativa de los estudiantes. Tal competencia se da en términos de conocer no sólo el código lingüístico, sino qué decir, a quién y cómo decirlo en determinada situación. Esta tiene que ver con aspectos como cuándo hay que hablar y cuándo guardar silencio y cuáles son las rutinas para tomar la palabra en una conversación.

Nuestro estudio confirma los resultados obtenidos por García (2005) en cuanto a que a los hablantes no nativos se les dificulta tomar la iniciativa en el manejo de la conversación, prefieren tomar el turno en un lugar apropiado para la transición antes que interrumpir y tienden a seguir estrictamente el tema de conversación. Se confirma también la tendencia, de acuerdo con Ambjoern (2008: 9), de que "el flujo insuficiente (pausas indeseadas) en la conversación se debe a la falta de práctica de los estudiantes a la hora de manejar los turnos".

Para estudios futuros, sería interesante profundizar específicamente en los patrones de conversación que rigen la primera lengua, pues podría tratarse de un fenómeno de transferencia negativa, que se ha estudiado bastante en fonología, pero no tanto en el discurso y menos aún en el análisis de la conversación (Calsamiglia y Tusón 1999; Briz 1998). Al respecto, en el caso de la lengua japonesa, podemos hacer referencia al estudio de Sato (citado en Chaudron 1988: 105), quien compara el manejo de turnos por parte de estudiantes asiáticos (chinos, japoneses y coreanos) y no asiáticos, para concluir que los primeros utilizan significativamente menos el mecanismo de autoselección. Noguchi (1997), por su parte, propone que, en general, los japoneses valoran más las reglas de proyección y protección de una imagen positiva que las reglas de mantenimiento de la conversación, mientras que hablantes de otras culturas, en este caso nativos del inglés, lo hacen a la inversa. Esto provoca que muchas veces los japoneses prefieran permanecer en silencio y perder el turno, aunque manejen de forma aceptable otros componentes de la lengua, como el morfosintáctico, el léxico y el fonológico.

Varios autores (Ambjoern 2008; García 2005) coinciden en que las clases de segundas lenguas están muy cargadas del enfoque pregunta-respuesta, siempre dirigido por el profesor y bastante predecible. Aún cuando solamente analizamos un caso particular, coincidimos con esta perspectiva y creemos que definitivamente incide de forma negativa en la dinámica conversacional, dado que no favorece la formación de los estudiantes en el manejo de estrategias discursivas que les permitan participar de forma apropiada y activa en la conversación.

Por último, siguiendo a Brenes Peña (2010), rescatamos también la necesidad de incorporar en el aula de español como segunda lengua el uso y estudio de transcripciones acordes con el nivel de manejo del idioma, de manera que los estudiantes puedan analizar cómo se estructura la conversación, incluyendo la alternancia de turnos de habla. Este análisis, creemos, favorecería la adquisición de estrategias para gestionar el turno y, por ende, conduciría a una mejor competencia conversacional. 


\section{Notas}

1. Esta corriente antropológica plantea que la competencia lingüística forma parte del conjunto de conocimientos y habilidades que componen la competencia comunicativa, que, a su vez, es parte de la competencia cultural (Calsamiglia y Tusón: 1999).

2. En términos generales, la dimensión interlocutiva hace referencia a cómo los interlocutores comprenden y emiten los enunciados y, mediante ello, ejecutan actos de habla particulares (Alcaraz y Martínez: 2004).

3. Siguiendo los postulados de Briz (2000), las intervenciones no numeradas constituyen momentos de la conversación donde hubo un cambio de voz pero no de turno, dado que la intervención no fue reconocida como tal por ninguno de los interlocutores.

\section{Bibliografía}

Albelda Marco, Marta y María José Fernández Colomer. 2006. "La enseñanza de los registros lingüísticos en E/LE. Una aplicación a la conversación coloquial". Revista de Didáctica. Marco ELE. (agregar número y volumen de la publicación): 2-31.

Alcaraz Varó, Enrique y María Antonia Martínez Linares. 2004. Diccionario de Lingüística Moderna. Barcelona: Ariel.

Ambjoern, Lone. 2008. "Enseñanza y aprendizaje de la competencia conversacional en español: planteamientos del problema y propuestas de solución". RedELE. Revista electrónica de didáctica / español lengua extranjera. (13): 1-15. http://dialnet.unirioja. es/servlet/articulo?codigo=2801045. Consulta: Agregar fecha.

Brenes Peña, Ester. 2010. "Interrupción y (des) cortesía. Algunas reflexiones en torno a la enseñanza y adquisición de las funciones estratégicas de la interrupción en las clases de L2.” En: Orletti, Franca y Laura Mariottini (eds), 721-742.

Briz Gómez, Antonio. 1998. El español coloquial en la conversación. Barcelona: Ariel.

2000. Turno y alternancia de turno en la conversación. Revista Argentina de Lingüística. (16): 9-32.

Brown, Gillian y George Yule. 1993. Análisis del discurso. Madrid: Visor.

Calsamiglia Blancafort, Helena y Amparo Tusón Valls. 1999. Las cosas del decir. Barcelona: Ariel.

Cestero Mancera, A. M. 2000. El intercambio de turnos de habla en la conversación. Análisis sociolingüístico. Alcalá de Henares: Servicio de publicaciones de la Universidad de Alcalá. 
Chaudron, C. 1988. Second Language Classrooms: Research on Teaching and Learning. Cambridge: Cambridge University Press.

García García, Marta. 2005. "La competencia conversacional en los estudiantes de español como lengua extranjera". Lingüística en la Red. (2):1-26. http://www.linred.es/ numero2.htm. Consulta: agregar fecha de consulta.

Grande Alija, Francisco Javier. 2005. "La cortesía verbal como reguladora de las interacciones verbales”. En: ASELE, Actas XVI: 332-342. Centro Virtual Cervantes. http://cvc. cervantes.es/ensenanza/biblioteca_ele/asele/pdf/16/16_0330.pdf. Consulta: Agregar fecha de consulta.

Gumperz, John y Hymes, Dell. 1972. Directions in sociolinguistics. The ethnography of communication. Nueva York: Basil Blackwell.

Kerbrat- Orecchioni, Catherine. 1996. La conversation. París: Armand Colin.

Noguchi, R. R. 1987. 'The Dynamics of Rule Conflict in English and Japanese Conversation'. International Review of Applied Linguistics in Language Teaching (IRAL). 15 (1): 15-23.

Orletti, Franca y Laura Mariottini (eds). 2010. Actas del V Coloquio EDICE (Des) cortesía en español. Espacios teóricos y metodológicos para su estudio. Roma-Estocolmo: Universitá degli Studi Roma Tre-EDICE.

Saville-Troike, Muriel. 1982. The ethnography of communication. An introduction. Londres: Basil Blackwell.

Shrum, Judith y Eileen Glisan. 1994. Teacher's Handbook. Boston: Heinle and Heinle Publishers. 
